\title{
Signal Processing for NDE
}

\author{
Masoud Vejdannik ${ }^{\mathbf{a}}$, Ali Sadr ${ }^{\mathbf{b}}$, Victor Hugo C. de Albuquerque ${ }^{\mathbf{c}}$, João Manuel R.S. Tavares ${ }^{\mathrm{d}}$ \\ a, b School of Electrical Engineering, Iran University of Science \& Technology (IUST), Narmak, \\ Tehran 16844, Iran. Email: masoud.vejdannik@gmail.com; sadr@iust.ac.ir \\ c Programa de Pós Graduação em Informática Aplicada, Universidade de Fortaleza (UNIFOR), \\ Fortaleza, Ceará, Brazil. Email: victor.albuquerque@unifor.br \\ d Instituto de Ciência e Inovação em Engenharia Mecânica e Engenharia Industrial, Faculdade de \\ Engenharia, Universidade do Porto, Porto, Portugal. Email: tavares@,fe.up.pt
}

\begin{abstract}
:
Nowadays, testing and evaluating of industrial equipment using nondestructive tests, is a fundamental step in the manufacturing process. The complexity and high costs of manufacturing industrial components, require examinations in some way about the quality and reliability of the specimens. However, it should be noted, that in order to accurately perform the nondestructive test, in addition to theoretical knowledge, it is also essential to have the experience and carefulness, which requires special courses and experience with theoretical education. Therefore, in the traditional methods, which are based on manual testing techniques and the test results depend on the operator, there is the possibility of an invalid inference from the test data. In other words, the accuracy of conclusion from the obtained data is dependent on the skill and experience of the operator. Thus, using the signal processing techniques for nondestructive evaluation (NDE), it is possible to optimize the methods of nondestructive inspection, and in other words, to improve the overall system performance, in terms of reliability and system implementation costs.

In recent years, intelligent signal processing techniques have had a significant impact on the progress of nondestructive assessment. In other words, by automating the processing of nondestructive data and signals, and using the artificial intelligence methods, it is possible to optimize nondestructive inspection methods. Hence, improve overall system performance in terms of reliability and Implementation costs of the system. This chapter reviews the issues of intelligent processing of nondestructive testing (NDT) signals.
\end{abstract}

\section{Introduction}

Nondestructive testing is an integral part of modern technology and has become a critical component of the engineering. The use of this technology in analyzing systems in terms of safety, reliability and mission guarantees is vital. Today, the strategic sectors in defense, space, aeronautics, information technology, electronics, nuclear, components of the power plant, railways and roads, consumer industries, material processing, conservation, restoration and certification of cultural heritage, use the nondestructive test as a worthwhile item. 
Identification of material defects is one of the most important applications in nondestructive testing. Regarding to the stages of manufacturing of engineering materials, occurrence of various abnormalities within them is possible. Depending on the considerations of the final operation, some of these defects and anomalies may be critical and complicated, and therefore their identification and classification is of great importance. Some of the methods used for this test include ultrasound testing, visual inspection, electromagnetic or eddy current testing, radiography and magnetic particle testing. Identifying the defects which cause to the catastrophic failures, and so are economically costly and include life-threatening hazards, is a clear advantage of the using nondestructive testing. Hence, using these test methods will have many advantages. The implementation of any inspection system involves costs, but often effective use of appropriate inspection methods will result in significant financial savings.

Despite the many advantages that the use of nondestructive testing results in identifying defects, the identification and classification of defects is often controversial, because the type of defects and their diagnosis are largely dependent on user experience and knowledge. The ability of the human eye to distinguish meaningful patterns, after a period of training and experience, is unsurpassed. However, an overwhelming flow of data reduces the efficiency of the eye in extracting the relevant information to make the exact decision. In addition, in some applications of the NDT, we seek to characterize the physical and mechanical properties of materials. For example, Vejdannik and Sadr in (Vejdannik and Sadr 2016a, Vejdannik and Sadr 2016b, Vejdannik and Sadr 2015, Vejdannik and Sadr 2016c) processed and classified the backscattered ultrasound signals which have been acquired from the specimens of nickel alloy, in order to investigate the microstructural properties of these materials. Certainly, this application is not feasible in the traditional way, or at least scanning electron microscopes should be used, which is time-consuming and costly. Therefore, traditional nondestructive testing methods based on empirical and innovative methods for identifying the pattern will lead to numerous problems in terms of cost, duration, and consequent unpredictable analysis, and thus will lead to contradiction and mismatch in the results.

Various signal processing tools, statistical methods and soft computing are introduced to solve the existing problems. The use of these processing tools for evaluation and classification process, should address the problems mentioned in the previous section by improving the measurement accuracy and reducing the load imposed on the operator. In this way, the user can focus more effectively on the outcome of the evaluation. The main advantages of using this computational approach is to develop a decision support system for accurately identifying and classifying flaws and defects, which will provide a standardized and impartial performance.

Section 2 briefly reviews the role of signal processing in NDT. In section 3, we will discuss about the fundamental components of NDT inspection system and issues of signal processing in a typical automated NDE systems. Finally, section 4 summarizes this chapter, presents conclusions, and describes the future directions for this topic. 


\section{Applications of signal processing to NDT}

In the first years of the emergence of nondestructive assessment systems, the researchers' efforts to improve NDT were only to increase the accuracy and resolution of inspection equipment to identify smaller defects. In other words, most of the works in the NDT domain were focused only on transducers and electrical equipment (analog signal processing). But in recent years, most of the researches and industrial applications have focused on the intelligent processing and automated processes that improve the reliability and content of information derived from the traditional methods. Many of these studies relate to the use of signal processing concepts, which have already been used successfully in other engineering areas such as radar, sonar, and geophysics.

A discrete time signal or time series is a set of observations that take place in a specified time sequence. The key characteristic of the time series is that the observations (in other words, samples), are in order of time and the adjacent samples are correlated (Manolakis et al. 2000).

To have a better observation of the relationship between signal samples consisting of sequences of length $l$, we plot the points $\{x(n), x(n+1)\}$ for $0 \leq n \leq N-1-l$ Where $N$ denotes the length of the signal data. This figure is known as the scattering plot. Figure 1, depicts a backscattered ultrasound signal and two associated scattering plots, which represent the correlation between adjacent samples. It can be seen that for adjacent samples, changes in the data points occur near a straight line. This implies the high correlation between the adjacent samples, because each sample is followed by another sample with an almost identical amplitude. In contrast, samples that are spaced at 20-point spacing are less correlated, because data points are randomly distributed in the scattering plot (Vejdannik and Sadr 2016a, Vejdannik and Sadr 2016b, Vejdannik and Sadr 2015, Vejdannik and Sadr 2016c).

When successive observations of the series are dependent, we may use past observations to predict future values. If the prediction is accurate, it is said that the series is a deterministic series. However, in many practical cases, we can't predict the time series exactly. Such series are called random or stochastic, and their degree of predictability is determined by the dependence between successive observations (Manolakis et al. 2000).

Signal processing is a well-known engineering term that can be defined in a broad sense as a transform that converts the signal data into useful information using a digital computer (Figure 2(a)). According to the above, it is observed that most of the theoretical results of the deterministic signal processing are inadequate to deal with NDT domain issues. Indeed, in many applications, the associated signal appears to be somewhat randomized, in the sense that the re-run of the test process does not result in the same observation. This is due to the disturbance that the user can not completely overcome. This disturbance can be of different types: noise or distortion effect applied on received signals, errors associated with measuring devices, and so on. Therefore, a state of confusion and uncertainty arises in the observations. The theory of probability and random 
processes provide an appropriate framework which can model this lack of knowledge. Finally, using these methods (statistical and intelligent signal processing), it is possible to take steps toward automation, or in other words, increase in the accuracy of results.

(a)

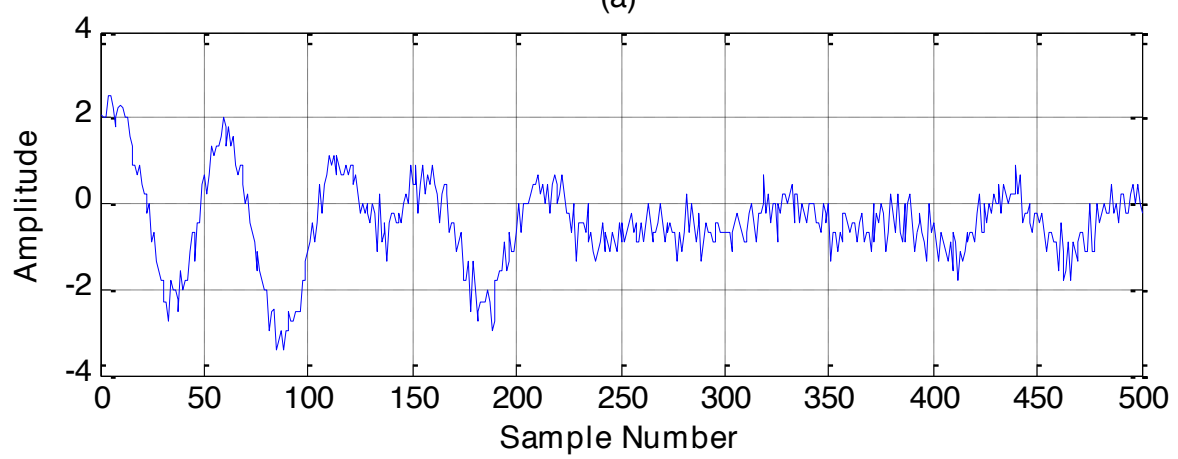

(b)

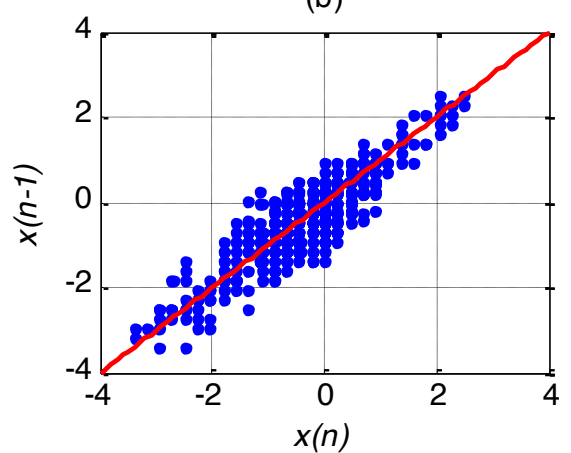

(c)

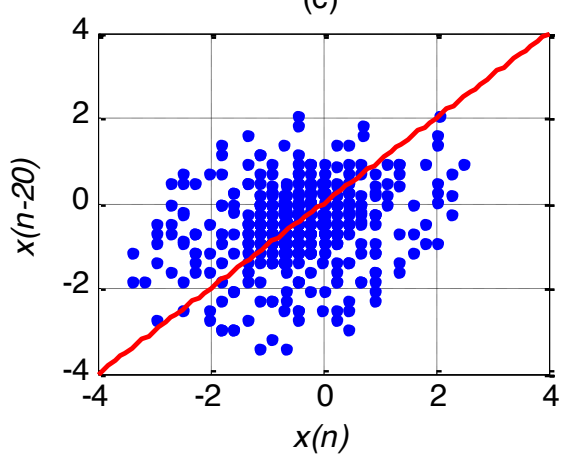

Figure 1: (a) A typical backscattered ultrasound signal. (b) Scattering plot for the adjacent samples. (c) Scattering plot for samples that are spaced at 20-point spacing

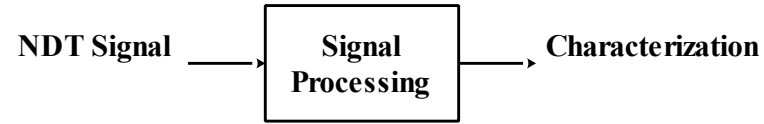

(a)

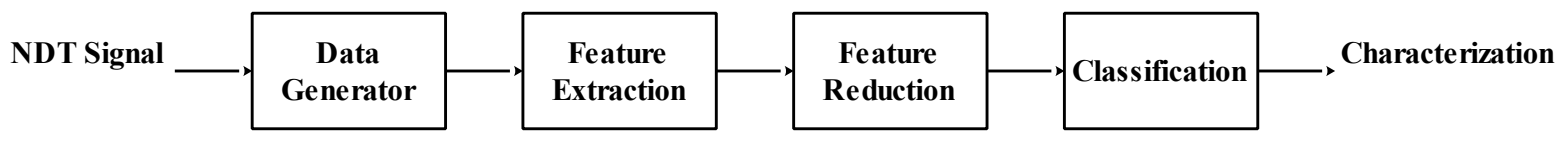

(b)

Figure 2: (a) NDT signal processing system, (b) Four basic building blocks of NDT signal processing system

In this chapter, in order to automate the NDE process in different applications, such as automatically characterize mechanical and metallurgical properties of materials, defects and heterogeneities within them, as well as determine the state and life duration of industrial equipment, we are going to review different component parts of nondestructive signal processing 
and classification system (Figure 2(b)). This system consists of four basic blocks: data generator (preprocessing), feature extraction, feature reduction and classification. The role of feature extraction step is to generate a relevant set of parameters that can reveal the true nature of the context data set, which consists of a large number of data (signals). The purpose of reducing features is to select the most relevant features for recognizing patterns. Indeed, this step will reduce the computational requirements. Also, the classification step helps in deciding that each instance is belong to which class.

By analyzing the various applications of intelligent signal processing techniques in the NDT domain, it can be seen that many problems in this domain are solved by the signal processing. The advantages of using signal processing for NDE include (Singh and Udpa 1986):

- Improved inspection reliability

- Improved detection of defects

- Improve the characterization of defects (Nunes et al. 2013)

- Generating useful information for quantifying the lifetime of structures (e.g. mechanical properties of materials) (Albuquerque et al. 2015)

- Generating information related to the processes. For example, processes such as thermal aging and phase transformation are monitored and controlled by NDT sensors and signal processing.

\section{Nondestructive inspection systems}

The way in which NDT signals are acquired, makes a significant contribution to the overall success of the signal processing system (automated nondestructive inspection system). Failure to pair the characteristics of the signal acquisition system with the transducer can lead to irretrievable loss of information, so that subsequent attempts to recover data through signal processing methods will not be effective. For example, most ultrasound inspection equipment suffers from a low signal-tonoise ratio. This effect is caused by issues such as noise generated by acoustic and electronic sources (Chen et al. 1999). This is much more complicated about applications that use the backscattered ultrasound signals.

Therefore, critical issues in obtaining a signal, such as sampling frequency and the number of quantization levels, should be tested carefully. If signals are bandwidth restricted, the WhittakerShannon sampling theory dictates that the signal must be sampled at a rate equal to at least twice the highest frequency component of the signal. Failure to follow this rule can lead to an aliasing error, which can corrupt the signal information. In typical applications, signals are sampled at a rate of 2 to 5 times the Nyquist rate to minimize aliasing effects.

The limited number of quantizer bits leads to SNR degradation. It can be shown (Oppenheim and Scafer 2010) that for a uniform quantizer with b bits, SNR is obtained by:

$$
S N R=\left(6.02 b+10.79+10 \log _{10} \sigma_{x}\right) d B
$$


Where $\sigma_{x}$ is the signal variance. This relationship states that when the number of quantum bits increases, the SNR increases by about $6 \mathrm{~dB}$.

Therefore, an essential requirement is to design a processing system that maximizes the capability and performance of the nondestructive inspection system. In the following, it is attempted to find out the advantages and disadvantages of various methods of intelligent signal processing in the NDT by reviewing the researches carried out in this topic.

\subsection{Intelligent processing of NDT signals}

A signal processing system is called intelligent if it can extract the maximum information at any time from its input signal, in a non-stationary environment with unknown statistical characteristics. To enable such capability, the system must be able to identify, exploit and process a series of features. These features are: nonlinearity, adaptivity, robustness, among others (Haikeyin 2001). In order to provide such requirements, inevitably, along with various signal processing tools, we must use artificial intelligence and soft computing (a set of computational methods including neural networks, fuzzy logic, and mmetaheuristic optimization algorithms such as genetic algorithm).

So far, we have become familiar with the advantages of using computer-based systems in analyzing nondestructive test data rather than manual analyzes that always involve human error in its results. Now, it's worthwhile to compare soft and hard computing to find out the advantages and disadvantages of using these methods in analyzing NDT data and signals. In Table 1, a comparison is made between the advantages and disadvantages of soft computing and hard computing.

It can be seen from Table 1 that in many cases associated with NDT data, the use of hard computing is not feasible or there will be no satisfactory results. For example, consider the ultrasound testing case, the most important issues and problems with these signals are ambiguity, incompatibility and high sensitivity (sensitivity to confusion in the data). For example, when faced with the delamination defects (Simas Filho et al. 2013), or defects caused by a row of holes (for example, rivet holes in thin metal sheets (Liu et al. 2013), reflected echoes from these defects interfere together and led to a condition of ambiguity and vagueness in making the right decision. In these conditions, fuzzy rules can create the required compatibility with the ambiguity and sensitivity by generating a fuzzy model. On the other hand, in many cases, due to the special characteristics of material and the impossibility of making an appropriate coupling between the surface of the specimen and the probe, a large amount of noise is integrated over the received signals. However, by generalizing the neural networks through learning, and due to the distributed structure of these networks, they can significantly deal with the sensitivity of these signals. So far, there have been many reports of the ability of neural networks to deal with signal noise and lack of disturbance on the output (the decision has been taken) of these networks (Farley et al. 2012). 
Table 1: Comparison of soft and hard computing

\begin{tabular}{ll}
\multicolumn{1}{c}{ Hard Computing } & \multicolumn{1}{c}{ Soft Computing } \\
\hline Input values must be accurate and definite. & It can also work with vague and noisy data. \\
\hline The resulting answers are accurate. & The resulting answers are approximate. \\
\hline Calculations are done sequentially. & Parallel processing and computing is possible. \\
\hline The problem is solved algebraically. & $\begin{array}{l}\text { A stochastic condition is involved in solving } \\
\text { problems. }\end{array}$ \\
\hline Can use only two valued logic. & Can use multiple valued logic. \\
\hline $\begin{array}{l}\text { To solve problems, an exact analytical model } \\
\text { and high computing time are required. }\end{array}$ & $\begin{array}{l}\text { The main model is formed by the help of the } \\
\text { human mind. }\end{array}$ \\
\hline $\begin{array}{l}\text { To solve specific issues, reprogramming is } \\
\text { required. }\end{array}$ & $\begin{array}{l}\text { Only existing apps should be upgraded. } \\
\text { Inaccuracies are considered inappropriate. }\end{array}$ \\
$\begin{array}{l}\text { In order to gain more flexibility, they bring } \\
\text { approximation and inaccuracy into the } \\
\text { problem. }\end{array}$ \\
\hline
\end{tabular}

One of the other problems we are encountered in analyzing NDT data is the high dimensionality and inconsistency; for solving such problems, the genetic algorithm (evolutionary computation) can be a very convenient and efficient method (Su et al. 2015, Acciani et al. 2010). However, it should be noted that the theory of rough sets can be used to reduce the rules. Another important note is that hard computing methods cannot adapt to the complexity of nondestructive tests. For example, characterizing the type of defect among several possible defects, makes such a complexity so that hard computing methods fail to analyze them (Nunes et al. 2013, Simas Filho et al. 2013, Farley et al. 2012, Su et al. 2015, Baker and Windsor 1989, Baker and Windsor 1992, Margrave et al. 1999, Santos and Perdigão 2001). While the proper combination of methods, such as hybrid neuro-fuzzy networks, can provide an appropriate and efficient solution. For example, Vejdannik and Sadr in (Vejdannik and Sadr 2016b) and (Vejdannik and Sadr 2016c) optimized the PNN (Probabilistic Neural Network) and RBF (Radial Basis Function) neural networks using the Bees Algorithm and so they were able to improve the accuracy of classifying ultrasound signals for characterizing the microstructures within the materials.

Other works of our research group evaluated the potentials of several machines learning techniques combined with various methods of feature extraction to characterization automatically the phase transformations using ultrasonic signal processing. Overall, the results achieved evidence that adopted algorithms were robustness in terms of accuracy and processing time. For example, Silva et al. (Silva et al. 2016a, Silva et al. 2016b, Silva et al. 2016c, Silva et al. 2016d) applied an induced magnetic field on a duplex stainless steel to characterize its microstructures, Albuquerque et al. (Albuquerque et al. 2010), Freitas et al. (Freitas et al. 2011), Silva et al. (Silva et al. 2009) analyzed the behavior of an ultrasound signal to evaluate the spinodal decomposition mechanism in duplex stainless steels under different aging thermal conditions. Albuquerque at al. (Albuquerque et al. 2012, Albuquerque et al. 2010a) applied the concepts of NDT-based signal processing to 
characterize the mechanical properties two novel materials, such as Nb-bearing nickel based superalloy weld overlays, and CuAlBe shape memory alloy, as well as in plain carbon steel (Freitas et al. 2010). In addition to one-dimensional signal processing, our group has used image analysis and processing techniques to perform non-destructive inspection in metallic and composites materials, as can be seen in (Albuquerque et al. 2011, Albuquerque et al. 2009, Albuquerque et al. 2010, Albuquerque et al. 2008, Papa et al. 2013, Durão et al. 2013, Durão et al. 2012, Papa et al. 2012, Durão et al. 2010, Albuquerque et al. 2010b, Kuncheva 2004, Cau et al. 2006).

\subsection{Preprocessing}

One of the most important tasks and reasons for using signal processing algorithms in nondestructive evaluation of materials is the automatic characterization of defects within them. Before proceeding to continue the discussion, it is necessary to overview the general scheme for characterizing the flaws (Figure 3). The energy is transmitted through a transducer into the material or the test object and interacts with the defect/material. The intensity of this interaction is a function of the type of excitation

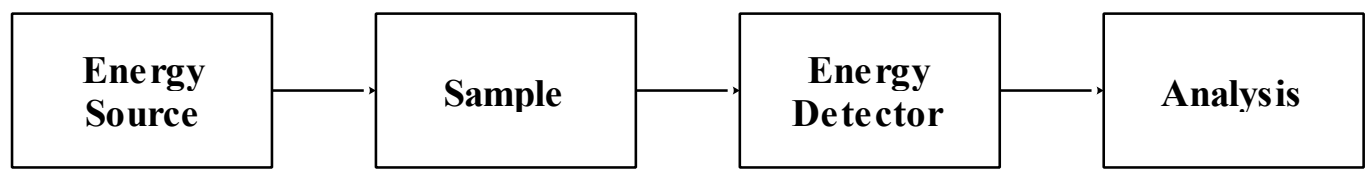

Figure 3: General model for NDE inspection

source and the properties of the material under test. On the other hand, the response signal that reflects the interaction of energy/material is received through a receiver transducer and displayed as the output of system. An ultrasonic test setup is a good example for demonstrating this scheme (Figure 4).

The main challenge in this NDT scenario is to estimate the characteristics of defects and properties of the material with having the excitation and response signals. Most of the data recording and analyzing methods are very simple and basic, and thus have a low reliability. The use of signal processing can minimize the user dependencies by automatically capturing and analyzing data. It can therefore improve the reliability of the NDT inspection system.

One of the most important goals of using signal processing in the NDT is to improve the separation capability for inspecting isotropic and uniform materials, as well as materials which led to the complexity when tested by the traditional NDT techniques. This includes materials with large particles, impedance mismatching and so on. For example, Nunes et al. (Nunes et al. 2013, Albuquerque et al. 2015) to investigate the microstructural properties of nickel alloy, which was exposed to thermal aging for 10, 100 and 200 hours at 650 and 950 degrees Celsius, analyzed the backscattered ultrasound signals. Due to the microstructural changes and phase transformation in the specimens, the backscattered signals were contaminated with noise. 


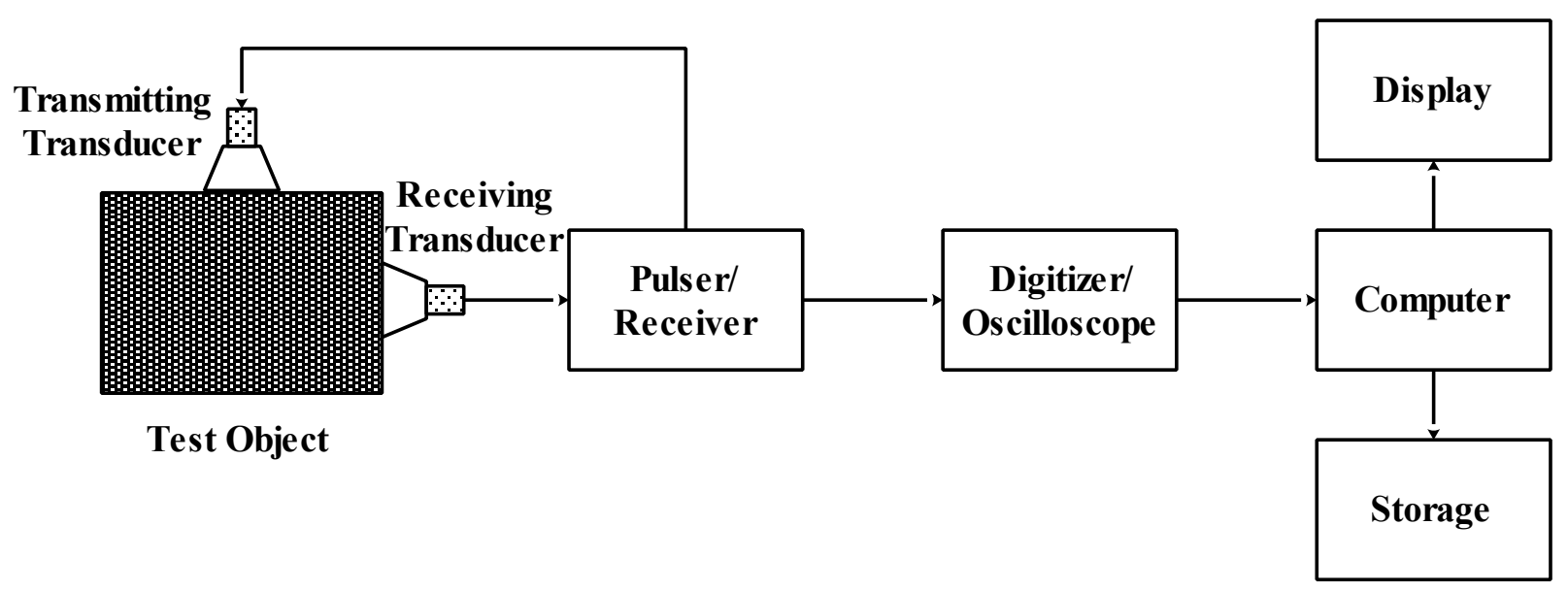

Figure 4: Basic ultrasonic test setup

NDT signals are often corrupted by the noise and artifacts which are generated by the transducers, tools, and other components inside the measuring equipment. This effect can be minimized by a proper signal conditioning. When the noise spectrum is accurately characterized and is outside the signal spectrum, the SNR can be improved by designing a simple filter. In practice, both of these simple methods are inadequate in themselves and so, more complicated methods are required. For example, Vejdannik and Sadr (Vejdannik and Sadr 2016) used the higher-order statistical methods in ultrasound signal processing which can implicitly remove the Gaussian noise in these signals.

\subsection{Features extraction methods}

A fundamental step in designing each signal classification system is the selection of a relevant set of features that can discriminate the signal in the feature space. A classification algorithm will always have somewhat identical results, but providing irrelevant features to it will not be able to reveal the true nature of the context data. Data should be reduced without losing information. Usually, in order to improve the performance and increase the computational efficiency, reducing the dimensionality of input data is desirable, and in many cases, this is achieved by applying a series of additional and appropriate transforms. Feature selection required to fulfill a fundamental criterion; features must retain all and only the significant information that is included in the data. This requirement has strong arguments about what transform should be used in the process of feature extraction. Finding the best features is a very important task and can usually be realized only through the process of trial and error.

Hence, an essential step in nondestructive inspection systems (especially in portable and inexpensive systems) is the feature reduction step. The main reason for generating (extracting) features using the transforms is that, if a proper transform is used, the redundant information will be removed from the original information. Redundant information is usually found in a set of information obtained by the measuring equipment. For example, the properties of backscattered 
ultrasound signals (these signals are random, non-linear, non-stationary and non-Gaussian) are such that the selection of features that contain the useful information from these signals is difficult and complicated. The advantage of using transforms is that the obtained information is relatively uncorrelated, thus there is no redundant information (Iyer et al. 2012).

Therefore, in choosing a feature extraction method for the NDT data, it is necessary to pay attention to these points: firstly, features should be selected that maintains critical information and discard any unnecessary data. Secondly, the method used should have a high energy packing capability in order to reduce the computational requirements and subsequently reduce time and costs. In Table 2, a comparison is made between the common feature extraction techniques used in the analysis of nondestructive tests.

So far, there have been many reports of the success of all these methods, but each of these methods has advantages and disadvantages that a more detailed examination of them can be useful and inspiring to achieve a more efficient and more robust system. One of the most important and essential issues which must be considered in the selection of feature extraction method, especially in nondestructive tests, is the computational complexity of the method used. Because most NDT inspection equipment are designed and manufactured to provide more mobility for the operator and can be used in various industrial situations (Simas Filho et al. 2013). Also, if the desired transform has a high energy packing efficiency, that is, the useful information of the signal is limited to a small number of coefficients, the implementation of the system requires less computational requirements, which can also be effective in speeding up and reducing costs.

Among these methods, the SVD transform has a strong capability in information packing, and thus, NDT data can be replaced by a smaller number of its singular values. However, vectors are not constant in SVD and K-L expansions. Indeed, they are application dependent and resulted from an optimization process. But this will increase the computational complexity. One can use the discrete Fourier transform that has less computational complexity, but instead it has a low energy packing capability. Also, less computational complexity can be provided by using the fast Fourier transform algorithm (Acciani et al. 2010, Kesharaju et al. 2014). DCT is a member of a family of transforms that uses a fast algorithm with relatively low complexity. Because of that DCT transforms the coefficients into a small domain, it provides an applicatory information packing efficiency for the NDT signals.

Table 2: Advantages and disadvantages of conventional feature extraction methods in NDE

Feature Extraction Methods

\begin{tabular}{lll}
\hline Karhunen-Loeve Transform & $\begin{array}{l}\text { - Features are completely } \\
\text { uncorrelated. }\end{array}$ & $\begin{array}{l}\text { - Depends on the application } \\
\text { - High computational } \\
\text { complexity }\end{array}$ \\
\hline Singular Value & - It has a very good packing & - It works on a fixed matrix. \\
Decomposition & efficiency. & So it is not suitable for \\
\hline
\end{tabular}




\begin{tabular}{|c|c|c|}
\hline & $\begin{array}{l}\text { - Basis vectors are the result } \\
\text { of an optimization process. }\end{array}$ & $\begin{array}{l}\text { working with adaptive } \\
\text { algorithms. } \\
\text { - Depends on the application. } \\
\text { - The relatively high } \\
\text { computational complexity } \\
\text { due to the optimization of } \\
O\left(N^{3}\right)\end{array}$ \\
\hline Discrete Fourier Transform & $\begin{array}{l}\text { - Moderate computational } \\
\text { complexity } O\left(N^{2}\right) \text { or } \\
\text { relatively low for fast Fourier } \\
\text { transform } O\left(\log _{2} N\right)\end{array}$ & $\begin{array}{l}\text { - It has a low data packing } \\
\text { efficiency }\end{array}$ \\
\hline Discrete Cosine Transform & $\begin{array}{l}\text { - A fast algorithm with } \\
\text { relatively low complexity } \\
O\left(\log _{2} N\right) \\
\text { - Very good packing } \\
\text { efficiency }\end{array}$ & $\begin{array}{l}\text { - It requires an extra } \\
\text { quantization step in the } \\
\text { output. }\end{array}$ \\
\hline Haar Transform & $\begin{array}{l}\text { - Multiresolution analysis tool } \\
\text { - Moderate Energy Packing } \\
\text { efficiency }\end{array}$ & $\begin{array}{l}\text { - Frequency response of Haar } \\
\text { filters is not ideal. }\end{array}$ \\
\hline Discrete Wavelet Transform & $\begin{array}{l}\text { - Multiresolution analysis tool } \\
\text { - Relatively high energy } \\
\text { packing efficiency } \\
\text { - A fast algorithm with } \\
\text { relatively low complexity } \\
O(N)\end{array}$ & $\begin{array}{l}\text { - Shift-variant } \\
\text { - Aliasing } \\
\text { - Oscillation }\end{array}$ \\
\hline
\end{tabular}

Among these methods, energy packing efficiency of Haar transform is rarely good, but instead provides multiresolution tools. However, wavelet transform can significantly improve the energy packing efficiency by choosing a proper mother wavelet. The wavelet transform has a very good localization property. In fact, the wavelet transform is used to find the moment at which an abrupt change occurs in the frequency domain at a given time.

Among the various methods used to extract features in the NDT signal processing, most success reports relate to the use of wavelet transform (Liu et al. 2013, Farley et al. 2012, Acciani et al. 2010, Barry et al. 2015, Crouch et al. 2015, Kesharaju et al. 2015, Duda et al. 2001, Vejdannik and Sadr 2016d). In fact, due to the properties of most NDT signals (these signals are non-stationary) which often led to the distribution of information at specific resolution levels, energy is often concentrated in a small number of samples. Although the DWT processing method is an efficient and effective method for analyzing non-stationary signals such as ultrasound signals (Figure 5 shows the approximate probability distribution for the five variables of the random process $x(t)$, with the same intervals. The unequal distribution of probabilities in these random variables indicates the variability of the probability distribution function in time, or in other words, the nonstationarity of the signal $x(t)$.), this method lacks the shift invariance property. Therefore, when 
the input signal is shifted, the energy of the wavelet coefficients varies considerably. The DTCWT processing method solves this problem by considering a complex structure (inspired by the Fourier transform).

As can be seen in Figure 6, variations in the average power of complex wavelet coefficients are very small compared to the average power of discrete wavelet coefficients. Therefore, the application of complex wavelet transform is more efficient to extract invariant features for the classification of NDT signals than discrete Wavelet transform (Vejdannik and Sadr 2016c).
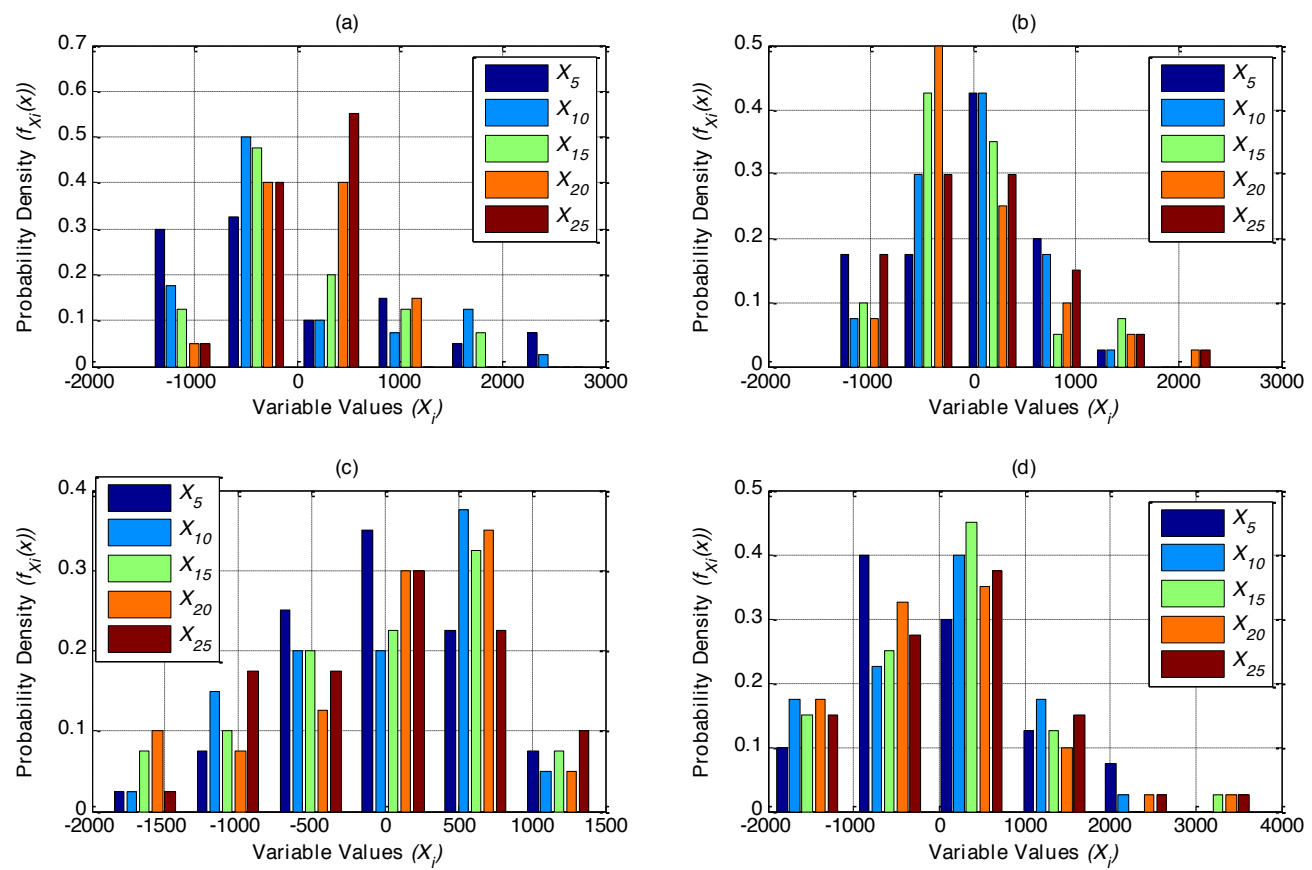

Figure 5: Approximate probability distribution for the five variables of the random process $\boldsymbol{x}(\boldsymbol{t})$ (an ultrasound signal in four different microstructural classes (Vejdannik and Sadr 2016c), with the same intervals - the unequal distribution of probabilities reveals that the signal is nonstationary). 

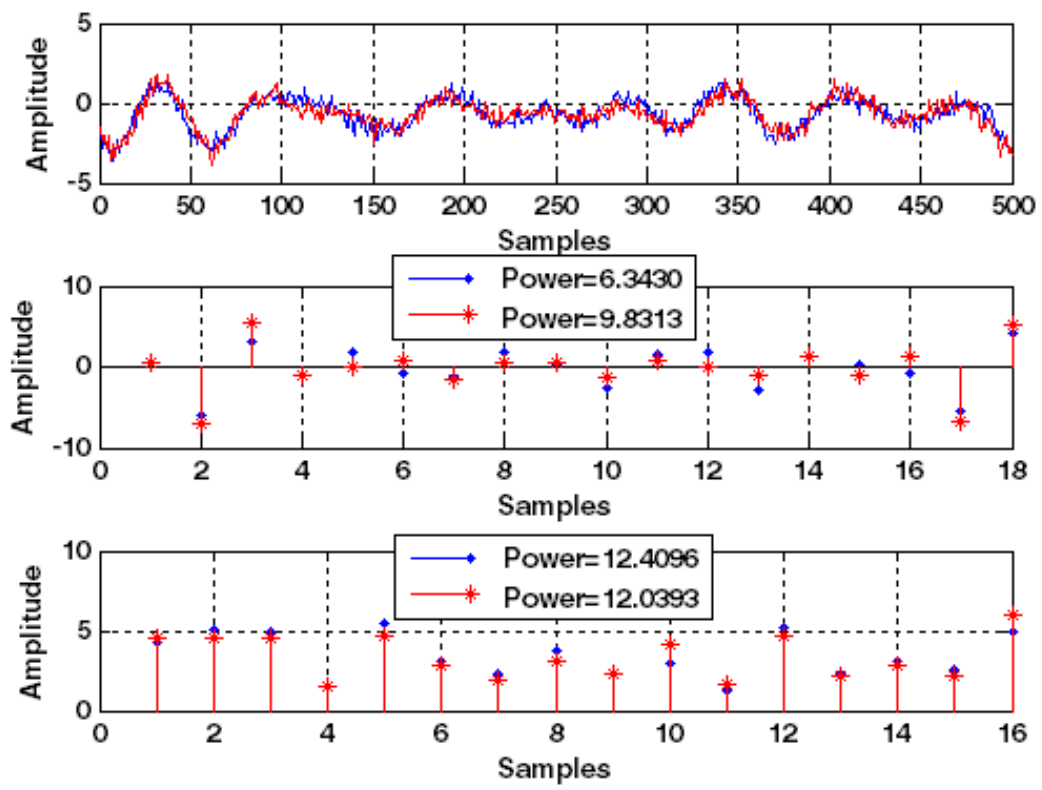

(c)

Figure 6: (a) Two ultrasound signals in two different classes (Vejdannik and Sadr 2016c). (b) Fifth scale detail coefficients of the (a) signals using DWT. (c) Fifth scale detail coefficients of the (a) signals using DTCWT.

On the other hand, NDT signals are inherently nonlinear, so conventional linear methods in the time and frequency domain cannot clearly characterize the complexity of such signals. For this purpose, Vejdannik and Sadr (Vejdannik and Sadr 2016a) used higher-order spectra analysis methods to detect the nonlinear relationship type and phase information between frequency components to characterize different phases in the process of phase transformation of Inconel 625 alloy. The motivation to use the higher-order spectra analysis methods are: (1) this method can eliminate Gaussian noise in the unknown spectral specifications. If a non-Gaussian signal is combined with a Gaussian noise, this noise can be eliminated using an HOStransform (this will increase the signal-to-noise ratio). (2) HOS methods maintain the phase information. For example in practice, it may sometimes some of the harmonic frequencies interact with each other and cause that summation/subtraction of their frequencies appears as a new frequency component in the power spectrum. (3) HOS methods can play a key role in identifying and characterizing nonlinearity in a system.

In addition to the above, scaling of the extracted features is also necessary, especially when the data covers different ranges. After scaling, the normalization of the data set is implemented, so all the inputs and target outputs have the same mean of zero and standard deviation of 1 . The goal of normalization is to ensure that all the features have an almost equal weight and large quantities will not prevail over other values.

\subsection{Feature reduction Methods}


One of the main issues in classification/pattern recognition (compatible with different NDT tests) is to select the appropriate range of features, which provides the independent or discriminant information required by the classifier for the correct classification of defects. Often, a large collection of potentially useful features is collected (feature extraction), and then the most appropriate and relevant features are selected by reducing the features (Figure 7). Indeed, all the extracted features are not relevant to the pattern recognition task. These features entail some kind of doubt and ambiguity on the problem, and consequently reduce the ability of identification (Song and Schmerr 1992).

Methods used in the analysis of nondestructive tests for feature reduction includes genetic optimization algorithms ( $\mathrm{Su}$ et al. 2015, Acciani et al. 2010), principal component analysis (Simas Filho et al. 2013, Cau et al. 2006), RS and DFA (Nunes et al. 2013). Among these works,

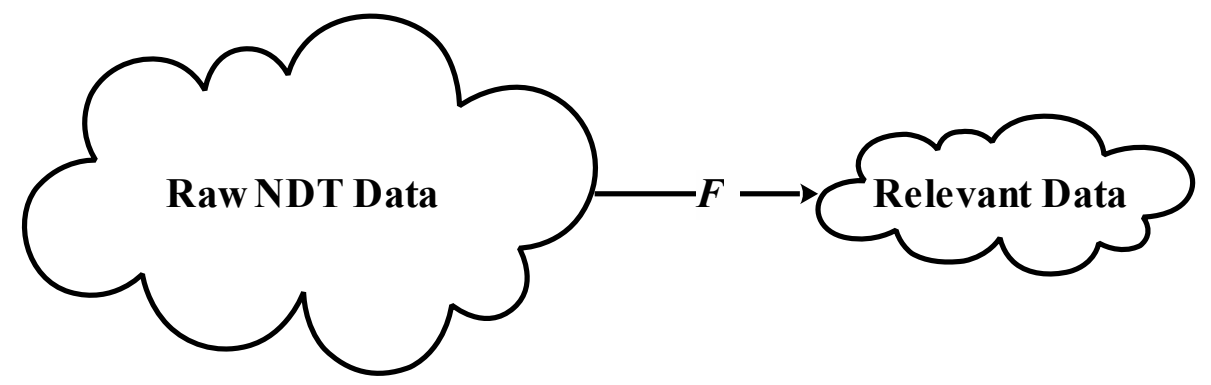

Figure 7: Mapping of raw NDT data into feature space

the RS and DFA algorithms have yielded poor results, but GA and PCA have brought successful results. Vejdannik and Sadr in (Vejdannik and Sadr 2016a) and (Vejdannik and Sadr 2015) used LDA to reduce the number of features in ultrasound signal processing and consequently ANOVA to select relevant coefficients.

In (Akram et al. 2014), three statistical methods PCA, LDA and ICA were used to reduce the number of NDT features and the results were compared with each other. Among the feature reduction methods applied in (Akram et al. 2014), ICA performed better than LDA and also LDA performed better than PCA. Indeed, PCA seeks to project features in the direction of the highest variability which are sorted by the eigenvectors. However, these directions do not necessarily provide the highest possible discrimination.

Like the PCA, LDA is looking for a linear combination of variables to reveal data in a better manner. However, the PCA does not distinguish between different classes, while the LDA explicitly tries to model the difference between the classes of data. Indeed, the LDA provides the highest discrimination between different classes and can therefore lead to a higher classification accuracy than the PCA method.

Besides, PCA and LDA methods explore the second-order statistics, and thus can only eliminate the statistical correlation between features. However, the ICA method uses higher-order statistics 
and therefore provides statistical independence. Indeed, higher-order statistics can reveal more significant information from the features. Hence, they can provide more discriminant features and consequently higher classification accuracy. The results obtained in (Akram et al. 2014), prove this.

\subsection{Classification Methods}

Classification is referred to a procedure in which we associate an unknown pattern (feature vector) to a class. This class can be one of the classes previously specified, or created by one or more new classes with a collection of unknown patterns.

The term supervised classification is referred to a procedure in which classifier design is performed using a set of training samples. In other words, label of each class is predetermined. Then, after designing the classifier, a set of test data is fed to the classifier, and the result of classifying each sample is determined.

In pattern recognition, the training samples and the corresponding classes are not always available. In this type of problems, there is a set of $x$ feature vectors and the purpose of problem is identifying the similarities and grouping or categorizing the similar vectors. This procedure is called unsupervised classification or clustering. A reasonable clustering algorithm gives clusters which in each cluster, samples are close together, while the similarity between clusters is as small as possible.

The final step in designing a decision support system (automated nondestructive inspection system) is to select a classifier so that it can, while maintaining high accuracy and speed (in comparison with existing methods and taking into account the requirements of each particular NDT application) and low computational requirements (with regard to the type of system, including portable and fixed), provides high reliability. Because the failure to identify some defects in industrial applications is catastrophic and leads to irreparable accidents.

The important point is that if we consider only the factor of accuracy and reliability, we cannot definitely announce the superiority of a classifier than others. But depending on the signal and the specific features that it has, each of these classifiers can achieve better result. For example, each of the MLP, RBF (Su et al. 2013), PNN, FCM (Karray and De Silva 2004), SVM, OPF (Nunes et al. 2013), LDA (Crouch et al. 2015) and Bayesian (Nunes et al. 2013, Bustillo et al. 2014a) classifiers can, in turn, have successful results by taking into account the characteristics of their classes. However, the reports obtained in (Nunes et al. 2013) demonstrate that the Bayesian classifier has a higher accuracy than the OPF, and the OPF classifier provides a higher accuracy than the SVM classifier. It should also be noted that several papers and experiments have reported the relative superiority of MLP classifiers to SOM, LVQ, SVM, and fuzzy methods in the classification of defects. 
Now, if we consider other factors such as computational requirements and speed, their fair comparison is feasible and provides useful results. Neural networks, due to their parallel structure, have a relatively high processing speed. However, they demand high computational requirements correspondingly (Bustillo et al. 2014ab). While OPF requires much less computational requirements while maintaining high processing speeds (Nunes et al. 2013). SVM is also far slower than this, and demands more computational requirement than OPF. On the other hand, the proposed neural networks in (Vejdannik and Sadr 2016b) and (Vejdannik and Sadr 2016c) provide much higher accuracy and speed. In these neural networks, four different approaches are presented for estimating the probability density function. Also, an optimization algorithm based on the Bees Algorithm is provided to set the parameters for these classifiers. The results obtained in these processing systems indicate that the intelligent NDT signal processing can greatly improve the reliability of nondestructive inspection systems by automating the process of characterizing defects and material properties.

\section{Conclusion}

Due to the rapid growth in the use of nondestructive testing to identify defects, the study of material properties, the growth of available data volumes, and the rapid increase in the methods of collecting these data, traditional and manual analysis of data is inefficient and computer-aided methods are accelerating. Novel signal processing techniques and artificial intelligence algorithms are being tested to solve various problems related to the nondestructive testing, especially ultrasound testing, so that they can provide a decision support system. This fast and user-friendly system can help industrial users and technicians to make decisions by reducing effort, time and, consequently the cost of classifying ultrasound test signals.

Indeed, in the traditional function, because the test results are dependent on the human user, the possibility of an invalid conclusion is very high. In other words, the accuracy of conclusion from obtained data is dependent on the skill and experience of the examiner. Thus, by the automated processing of NDT signals and using artificial intelligence techniques, it is possible to step up the optimization of nondestructive inspection methods, namely improving overall system performance, in terms of reliability and system implementation costs. In this regard, due to the random, non-linear and non-stationary properties of the NDT signals, AI methods and statistical signal processing techniques have been able to play an effective role in solving various NDT problems. However, in the NDE domain, less attention has been paid to the statistical processing of NDT signals than other engineering areas. Therefore, according to the properties of these signals, it is desirable to focus more on this branch in the future.

\section{Acknowledgments}

VHCA acknowledges the sponsorship from the Brazilian National Council for Research and Development (CNPq) via Grant No. 301928/2014-2. 
JMRST gratefully acknowledges the funding of Project NORTE-01-0145-FEDER-000022 SciTech - Science and Technology for Competitive and Sustainable Industries, co-financed by "Programa Operacional Regional do Norte" (NORTE2020), through "Fundo Europeu de Desenvolvimento Regional" (FEDER).

\section{References}

Vejdannik M, Sadr A (2016a) Automatic microstructural characterization and classification using higher-order spectra on ultrasound signals, Journal of Nondestructive Evaluation, 35:1

Vejdannik M, Sadr A (2016b) Automatic microstructural characterization and classification using probabilistic neural network on ultrasound signals. Journal of Intelligent Manufacturing. doi: 10.1007/s10845-016-1225-y

Vejdannik M, Sadr A (2015) Application of linear discriminant analysis to ultrasound signals for automatic microstructural characterization and classification, Journal of Signal Processing Systems, 83:3:411-421

Vejdannik M, Sadr A (2016c) Automatic microstructural characterization and classification using dual tree complex wavelet-based features and Bees Algorithm, Neural Computing and Applications, 28:7: 1877-1889

Manolakis D, Ingle V, Kogon S (2005) Statistical and adaptive signal processing. Artech House, Boston

Singh G, Udpa S (1986) The role of digital signal processing in NDT. NDT International 19:125132

Nunes T, de Albuquerque V, Papa J, Silva C, Normando P, Moura E, Tavares J (2013) Automatic microstructural characterization and classification using artificial intelligence techniques on ultrasound signals. Expert Systems with Applications 40:3096-3105

de Albuquerque V, Barbosa C, Silva C, Moura E, Filho P, Papa J, Tavares J (2015) Ultrasonic Sensor Signals and Optimum Path Forest Classifier for the Microstructural Characterization of Thermally-Aged Inconel 625 Alloy. Sensors 15:12474-12497

Chen J, Shi Y, Shi S (1999) Noise analysis of digital ultrasonic nondestructive evaluation system. International Journal of Pressure Vessels and Piping 76:619-630

Oppenheim A, Schafer R (2014) Discrete-time signal processing. Pearson, Harlow

Haikeyin (2001) Neural Networks : A Comprehensive Foundation =. Qing hua da xue chu ban she, Bei jing

Simas Filho E, Souza Y, Lopes J, Farias C, Albuquerque M (2013) Decision support system for ultrasound inspection of fiber metal laminates using statistical signal processing and neural networks. Ultrasonics 53:1104-1111

Liu S, Du C, Mou J, Martua L, Zhang J, Lewis F (2013) Diagnosis of structural cracks using wavelet transform and neural networks. NDT \& E International 54:9-18 
Farley S, Durodola J, Fellows N, Hernández-Gómez L (2012) High resolution non-destructive evaluation of defects using artificial neural networks and wavelets. NDT \& E International 52:69-75

Su L, Shi T, Du L, Lu X, Liao G (2015) Genetic algorithms for defect detection of flip chips. Microelectronics Reliability 55:213-220

Acciani G, Brunetti G, Fornarelli G, Giaquinto A (2010) Angular and axial evaluation of superficial defects on non-accessible pipes by wavelet transform and neural network-based classification. Ultrasonics 50:13-25

Baker A (1989) The classification of defects from ultrasonic data using neural networks: The Hopfield method. NDT \& E International 22:97-105

Margrave F, Rigas K, Bradley D, Barrowcliffe P (1999) The use of neural networks in ultrasonic flaw detection. Measurement 25:143-154

Santos J, Perdigão F (2001) Automatic defects classification - a contribution. NDT \& E International 34:313-318

de Macedo Silva E, Leite J, de França Neto F, Leite J, Fialho W, de Albuquerque V, Tavares J (2014) Evaluation of the Magnetic Permeability for the Microstructural Characterization of a Duplex Stainless Steel. Journal of Testing and Evaluation 44:20130313

de Macedo Silva E, Leite J, Leite J, Fialho W, de Albuquerque V, Tavares J (2016) Induced Magnetic Field Used to Detect the Sigma Phase of a 2205 Duplex Stainless Steel. Journal of Nondestructive Evaluation. doi: 10.1007/s10921-016-0339-7

Silva E, Marinho L, Filho P, Leite J, Leite J, Fialho W, de Albuquerque V, Tavares J (2016) Classification of Induced Magnetic Field Signals for the Microstructural Characterization of Sigma Phase in Duplex Stainless Steels. Metals 6:164

Silva E, Paula A, Leite J, Leite J, Andrade L, de Albuquerque V, Tavares J (2016) Detection of the Magnetic Easy Direction in Steels Using Induced Magnetic Fields. Metals 6:317

de Albuquerque V, de Macedo Silva E, Pereira Leite J, de Moura E, de Araújo Freitas V, Tavares J (2010) Spinodal decomposition mechanism study on the duplex stainless steel UNS S31803 using ultrasonic speed measurements. Materials \& Design 31:2147-2150

de Araújo Freitas V, Normando P, de Albuquerque V, de Macedo Silva E, Silva A, Tavares J (2011) Nondestructive Characterization and Evaluation of Embrittlement Kinetics and Elastic Constants of Duplex Stainless Steel SAF 2205 for Different Aging Times at $425^{\circ} \mathrm{C}$ and $475^{\circ} \mathrm{C}$. Journal of Nondestructive Evaluation 30:130-136

de Macedo Silva E, de Albuquerque V, Leite J, Varela A, Moura E, Tavares J (2009) Phase transformations evaluation on a UNS S31803 duplex stainless steel based on nondestructive testing. Materials Science and Engineering: A 516:126-130

de Albuquerque V, Silva C, Normando P, Moura E, Tavares J (2012) Thermal aging effects on the microstructure of Nb-bearing nickel based superalloy weld overlays using ultrasound techniques. Materials \& Design (1980-2015) 36:337-347 
de Albuquerque V, Melo T, de Oliveira D, Gomes R, Tavares J (2010) Evaluation of grain refiners influence on the mechanical properties in a $\mathrm{CuAlBe}$ shape memory alloy by ultrasonic and mechanical tensile testing. Materials \& Design 31:3275-3281

Freitas V, Albuquerque V, Silva E, Silva A, Tavares J (2010) Nondestructive characterization of microstructures and determination of elastic properties in plain carbon steel using ultrasonic measurements. Materials Science and Engineering: A 527:4431-4437

de Albuquerque V, Silva C, Menezes T, Farias J, Tavares J (2010) Automatic evaluation of nickel alloy secondary phases from SEM images. Microscopy Research and Technique 74:36-46

de Albuquerque V, de Alexandria A, Cortez P, Tavares J (2009) Evaluation of multilayer perceptron and self-organizing map neural network topologies applied on microstructure segmentation from metallographic images. NDT \& E International 42:644-651

DE ALBUQUERQUE V, FILHO P, CAVALCANTE T, TAVARES J (2010) New computational solution to quantify synthetic material porosity from optical microscopic images. Journal of Microscopy 240:50-59

de Albuquerque V, Cortez P, de Alexandria A, Tavares J (2008) A new solution for automatic microstructures analysis from images based on a backpropagation artificial neural network. Nondestructive Testing and Evaluation 23:273-283

Papa J, Nakamura R, de Albuquerque V, Falcão A, Tavares J (2013) Computer techniques towards the automatic characterization of graphite particles in metallographic images of industrial materials. Expert Systems with Applications 40:590-597

Durão L, Tavares J, de Albuquerque V, Gonçalves D (2013) Damage evaluation of drilled carbon/epoxy laminates based on area assessment methods. Composite Structures 96:576-583

Durão L, Gonçalves D, Tavares J, de Albuquerque V, Marques A (2011) Comparative analysis of drills for composite laminates. Journal of Composite Materials 46:1649-1659

Papa J, Falcão A, de Albuquerque V, Tavares J (2012) Efficient supervised optimum-path forest classification for large datasets. Pattern Recognition 45:512-520

Durão L, Tavares J, de Albuquerque V, Marques A, Magalhães A, Vieira A (2010) Tool Effects on Hybrid Laminates Drilling. Materials and Manufacturing Processes 25:476-481

De Albuquerque V, Tavares J, Durão L (2009) Evaluation of Delamination Damage on Composite Plates using an Artificial Neural Network for the Radiographic Image Analysis. Journal of Composite Materials 44:1139-1159

Kuncheva L (2014) Combining pattern classifiers. John Wiley \& Sons Inc

Cau F, Fanni A, Montisci A, Testoni P, Usai M (2006) A signal-processing tool for non-destructive testing of inaccessible pipes. Engineering Applications of Artificial Intelligence 19:753-760

Iyer S, Sinha S, Tittmann B, Pedrick M (2012) Ultrasonic signal processing methods for detection of defects in concrete pipes. Automation in Construction 22:135-148

Kesharaju M, Nagarajah R, Zhang T, Crouch I (2014) Ultrasonic sensor based defect detection and characterisation of ceramics. Ultrasonics 54:312-317 
Barry T, Kesharaju M, Nagarajah C, Palanisamy S (2015) Defect characterisation in laminar composite structures using ultrasonic techniques and artificial neural networks. Journal of Composite Materials 50:861-871

Crouch I, Kesharaju M, Nagarajah R (2015) Characterisation, significance and detection of manufacturing defects in Reaction Sintered Silicon Carbide armour materials. Ceramics International 41:11581-11591

Kesharaju M, Nagarajah R (2015) Feature selection for neural network based defect classification of ceramic components using high frequency ultrasound. Ultrasonics 62:271-277

Duda R, Hart P, Stork D (2001) Pattern classification.

Vejdannik M, Sadr A (2015) Application of Linear Discriminant Analysis to Ultrasound Signals for Automatic Microstructural Characterization and Classification. Journal of Signal Processing Systems 83:411-421

Song S, Schmerr L (1992) Ultrasonic flaw classification in weldments using probabilistic neural networks. Journal of Nondestructive Evaluation 11:69-77

Akram N, Isa D, Rajkumar R, Lee L (2014) Active incremental Support Vector Machine for oil and gas pipeline defects prediction system using long range ultrasonic transducers. Ultrasonics 54:1534-1544

Su L, Zha Z, Lu X, Shi T, Liao G (2013) Using BP network for ultrasonic inspection of flip chip solder joints. Mechanical Systems and Signal Processing 34:183-190

Karray F, De Silva C (2004) Soft computing and tools of intelligent systems design. AddisonWesley, Harlow

Bustillo J, Fortineau J, Gautier G, Lethiecq M (2014) Ultrasonic characterization of porous silicon using a genetic algorithm to solve the inverse problem. NDT \& E International 62:93-98

Bustillo J, Fortineau J, Gautier G, Lethiecq M (2014) Ultrasonic characterization of electrochemically etched porous silicon. Japanese Journal of Applied Physics 53:060308 